\title{
Cryoablation at Growing Myocardium: No Evidence of Coronary Artery Obstruction or Intimal Plaque Formation Early and Late after Energy Application
}

THOMAS KRIEBEL, M.D., * HANS-PETER HERMANN, M.D.,† HEIKE SCHNEIDER, M.D., * MAJA KROLL, M.D., * JAKOB SELLE, * ANNA OVERWAUL, * MATTHIAS SIGLER, M.D., * and THOMAS PAUL, M.D.*

From the * Department of Pediatric Cardiology and Intensive Care Medicine; and +Department of Cardiology and Pneumology, Georg-August-University Göttingen, Göttingen, Germany

Background: Animal studies and clinical observations have demonstrated that radiofrequency current application at growing myocardium may result in coronary artery obstruction. Recently, cryoenergy has emerged as an effective alternative to radiofrequency ablation of arrhythmogenic substrates in pediatric patients. Up to now, there has been a lack of experimental data concerning the effects of cryoenergy application at growing myocardium.

Methods: During general anesthesia, selective coronary angiography of the right and left coronary artery was performed in 10 piglets (age: 6 weeks, body weight: 14-18 kg). Subsequently, cryoenergy was delivered at $-75^{\circ} \mathrm{C}$ for 4 minutes using a 7-F catheter with a 6-mm-tip electrode at the atrial aspect of the tricuspid valve annulus in a posterior and lateral position. Additional cryoenergy lesions were induced via a retrograde approach at the lateral and posterior atrial and ventricular aspect of the mitral valve annulus, respectively. Five animals were restudied after 48 hours by coronary angiography and intracoronary ultrasound and in the remaining five piglets after 3 and 6 months, respectively.

Results: Selective coronary angiography and intracoronary ultrasound studies did not demonstrate any evidence for coronary artery obstruction after 48 hours, 3 months, or 6 months after cryoenergy application. In addition, histological examinations of the cryolesions after 48 hours and after 6 months did not demonstrate any intimal proliferations of the coronary arteries, respectively.

Conclusions: By means of the present study, we did not observe any affection of the coronary arteries after cryoenergy application at growing myocardium in young piglets. (PACE 2009; 32:1197-1202)

cryoablation, immature myocardium, coronary artery obstruction, intracoronary ultrasound, coronary angiography

\section{Introduction}

Electrophysiological study and radiofrequency ablation of supraventricular tachycardia substrates is an established therapy in pediatric patients with high efficacy and low complication rate. However, animal studies of our group have demonstrated $^{1,2}$ that radiofrequency current application at growing myocardium may result in coronary artery obstruction. Meanwhile, these experimental data were confirmed by reports of coronary artery obstruction and occlusion in adult as well as in pediatric patients following radiofrequency catheter ablation. $^{3-14}$ In addition, death of a 13year-old patient due to occlusion of the left coro-

Conflict of interest: None.

Address for reprints: Thomas Kriebel, M.D., Department of Pediatric Cardiology and Intensive Care Medicine, University Hospital, Georg-August-University Göttingen, Robert-KochStraße 40, D-37075 Göttingen, Göttingen, Germany. Fax: +49551-3922561; e-mail: tkriebe@gwdg.de

Received October 28, 2008; revised February 12, 2009; accepted April 26, 2009. nary artery (LCA) after successful radiofrequency ablation of a left-sided accessory pathway has been reported. ${ }^{15}$

Within the last years, cryoenergy has evolved as a safe and an effective alternative for catheter ablation of arrhythmogenic substrates in pediatric patients. However, in comparison to radiofrequency catheter ablation of supraventricular tachycardia in pediatric patients, lower success rates and higher recurrence rates have been observed in several studies. ${ }^{16-19}$ Due to specific features of this new technique, cryoadhesion and cryomapping, the risk of atrioventricular (AV) block can be minimized especially when targeting septal substrates. In addition, a study by Aoyama and coworkers $^{20}$ demonstrated no obstruction of the coronary artery after cryoablation in the coronary sinus close to the LCA in adult dogs. However, up to now, there has been a lack of experimental data concerning the effects of cryoenergy application at growing myocardium.

The aim of the present study was to assess coronary artery involvement by serial coronary angiography, intracoronary ultrasound studies,

(C)2009, The Authors. Journal compilation (C)2009 Wiley Periodicals, Inc. 
and histological examinations after cryoenergy application in piglets. The study was performed with an experimental design comparable with a previous study of our group using radiofrequency current $^{2}$ in which coronary obstruction had been demonstrated.

\section{Methods \\ Animals}

Ten piglets (German Landrace) with a mean age of 6 weeks and a mean body weight of $15.6 \mathrm{~kg}$ (range: 14-18 kg) were studied. The study had been approved by the local authority (Bezirksregierung Braunschweig, Germany, Ref. No. 509.42502/01-09.04). All experiments were performed under general anesthesia while administering midazolam, propofol, and ketamine intravenously with additional continuous inhalation of isoflurane. Vascular sheaths were placed in the femoral artery and vein by the percutaneous technique. Subsequently, heparin $(100 \mathrm{U} / \mathrm{kg})$ and cephazoline $(50 \mathrm{mg} / \mathrm{kg})$ were administered intravenously.

\section{Study Protocol}

Selective coronary angiography was performed in standard projections, i.e., right anterior oblique (RAO) $30^{\circ}$ angulation and left anterior oblique (LAO) $60^{\circ}$ angulation, respectively, to document coronary anatomy at baseline using 5-F Judkins left/right or 5-F Amplatzer left/right catheters (Cordis, Roden, Netherlands). Angiographies were digitally stored for off-line analysis (BV Pulsera, Philips, Amsterdam, Netherlands). Subsequently, a steerable $7-\mathrm{F}$ cryocatheter equipped with a 6-mm-tip electrode (Freezor ${ }^{\circledR}$ Xtra, Cryocath Technologies Inc., Quebec, Canada) was introduced antegradely into the right atrium and subsequently retrogradely into the left ventricle and left atrium, respectively. During sinus rhythm, the tip of the cryocatheter was placed at the respective AV groove with the aim to register appropriate bipolar local atrial and ventricular electrocardiograms. Under fluoroscopic guidance, cryoenergy application was performed with a target temperature of $-75^{\circ} \mathrm{C}$ for 4 minutes at the following locations:

(1) At the atrial aspect of the tricuspid valve annulus in a posterior and lateral position

(2) Via a retrograde approach at the posterior and lateral atrial aspect of the mitral valve annulus

(3) Via a retrograde approach at the posterior and lateral ventricular aspect of the mitral valve annulus

During cryoenergy application, selective coronary angiography was performed in order to control the local proximity of the ablation catheter to the coronary artery (Fig. 1). Each location of cryoenergy application was fluoroscopically recorded and documented. Thirty minutes after the final cryoenergy application selective coronary angiography of the left coronary artery (LCA) and right coronary artery (RCA) was repeated. Subsequently, the sheaths were removed, hemostasis was achieved, and the animals were extubated. Aspirin $(2-3 \mathrm{mg} / \mathrm{kg}$ ) was given afterwards on a daily basis to avoid thrombus formation at the ablation sites as it is standard practice in human ablation procedures at our institution to avoid thromboembolic events.
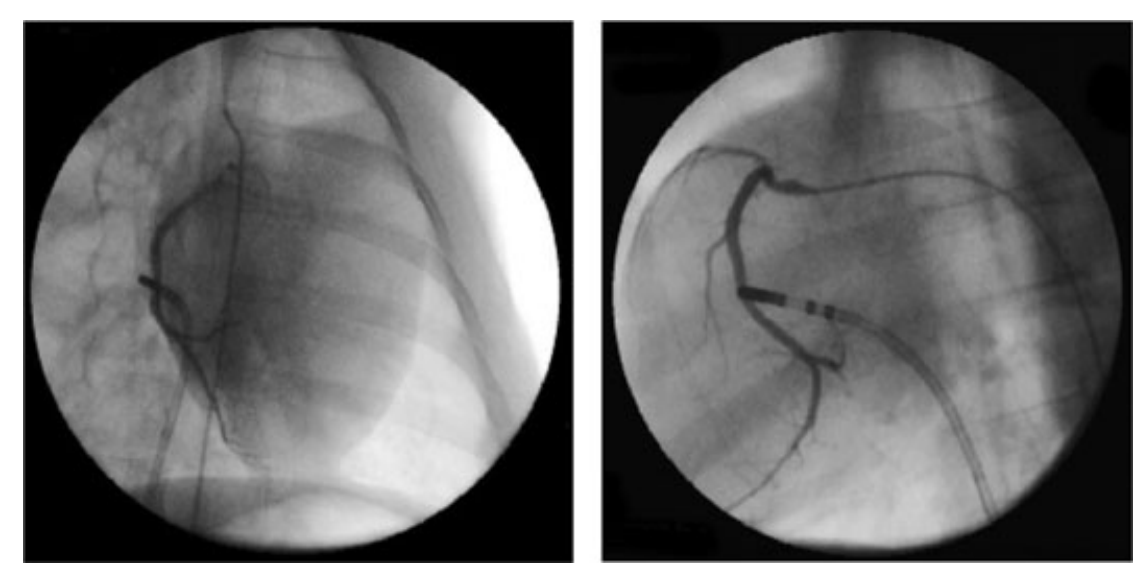

Figure 1. Cryoenergy application at the lateral tricuspid valve annulus (left panel $30^{\circ}$ right anterior oblique-projection, right panel $60^{\circ}$ left anterior oblique-projection) for 4 minutes at $-75^{\circ} \mathrm{C}$. Coronary angiography of the RCA was performed during cryoenergy application to demonstrate the proximity of the ablation catheter to the coronary artery. 


\section{Re-evaluation}

Forty-eight hours after cryoablation, five randomly selected animals (acute group) were restudied under general anesthesia as described earlier. Selective coronary angiograms of the LCA and RCA were repeated and again digitally stored for the off-line analysis. Subsequently, an intracoronary ultrasound study was performed. Before entering the vessels with the guide wire (see below), glycerol trinitrate (0.1-0.2 mg) and additional heparin $(50 \mathrm{U} / \mathrm{kg})$ were selectively administered into the RCA and LCA, respectively. Under fluoroscopic guidance, a 0.014-inch guide wire (HiTorque ${ }^{\circledR}$ Guide Wire, Guidant, Santa Clara, CA, USA) was advanced far distal into the RCA and into the left circumflex artery (LCx). Intracoronary ultrasound studies were performed using a 2.9-F ultrasound catheter (Jovus Avanar F/X, Volcano Therapeutics Inc., Brussels, Belgium) together with the respective ultrasound console (EndoSonics, Volcano Therapeutics Inc.). The ultrasound catheter was automatically retracted under fluoroscopic guidance in 1-mm intervals (Trak Back, EndoSonics Corporation, Rancho Cordova, CA, USA) from the peripheral vessel back to the ostium. Ultrasound images were continuously recorded and again digitally stored for the off-line analysis. Finally, animals were sacrificed for further histological work up.

The remaining five animals (chronic group) were restudied after 3 and 6 months as described above by selective coronary angiography and intracoronary ultrasound, respectively, and finally sacrificed after 6 months.

\section{Off-line Analysis}

Selective coronary angiograms and intracoronary ultrasound sequences were screened by two independent observers. Significant plaque for- mation or coronary artery obstruction was defined as $>30 \%$ diameter stenosis by quantitative coronary angiography measurements or thickening of the intima $>200 \mu \mathrm{m}$ by intracoronary ultrasound.

\section{Histological Analysis}

Tissue blocks containing the whole lesions with adjacent tissue including adherent coronary arteries were excised. After fixation and paraffin embedding, the blocks were sectioned at $5 \mu \mathrm{m}$ and staining was performed with hematoxylineosin and Elastica van Gieson using standard techniques.

\section{Results}

In all 10 piglets, no major complications were noted during the entire course of the study. During cryoablation, no ST-segment elevations or other major electrocardiograms changes were observed.

\section{Selective Coronary Angiographies}

Selective coronary angiographies of the RCA and LCA could be accomplished in all animals as planned. No evidence for coronary artery obstruction was noted after 48 hours as well as 3 and 6 months after cryoenergy application by the two independent observers (Fig. 2).

\section{Intracoronary Ultrasound}

Intracoronary ultrasound of the RCA and LCx could be performed in all animals of the acute group 48 hours after cryoenergy application and in all animals of the chronic group 3 and 6 months after cryoenergy application. Analysis of the intracoronary ultrasound studies with respect to intimal thickening and/or narrowing of the vessel diameter by the two independent investigators did
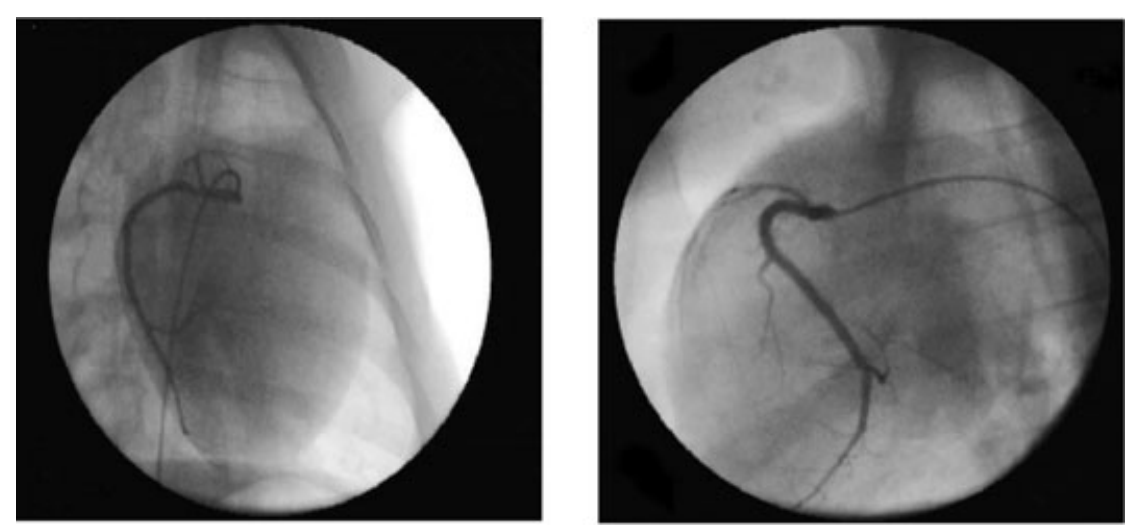

Figure 2. Coronary angiography of the RCA 6 months after cryoenergy application did not reveal any evidence for coronary artery obstruction (same animal and projections as in Fig. 1). 

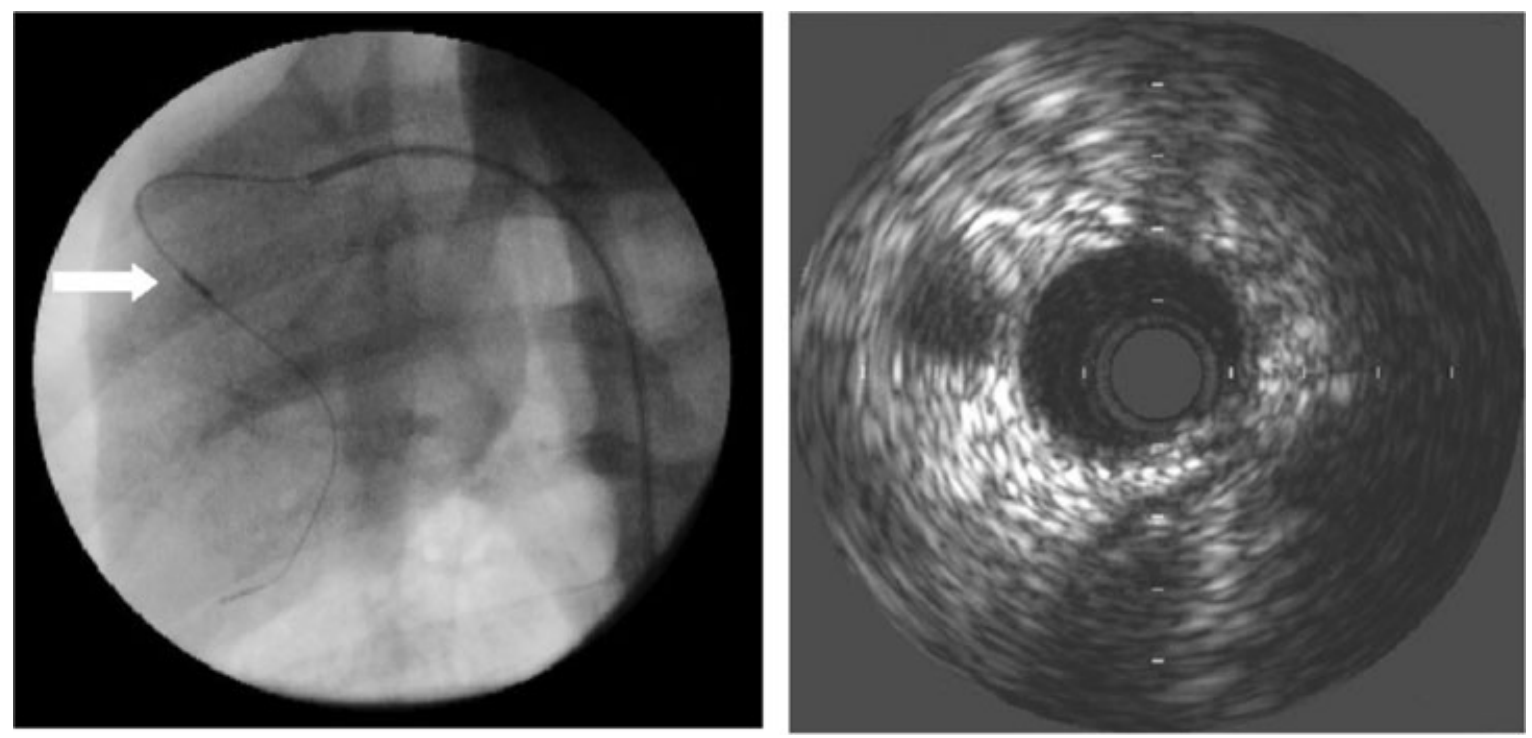

Figure 3. Intracoronary ultrasound study of the RCA 6 months after cryoenergy application (same animal as in Figs. 1 and 2). Left panel: Position of the ultrasound probe (arrow) within the vessel along the tricuspid valve annulus $\left(60^{\circ}\right.$ left anterior oblique projection). Right panel: Intracoronary ultrasound image of the RCA at the position displayed in the left panel. Inside the lumen of the coronary artery the ultrasound catheter is visible. A smooth endovascular surface without any evidence of plaque formation or stenosis can be seen.

not reveal any intimal plaque formation or stenosis (Fig. 3) in any of the animals, respectively.

\section{Histopathological Analysis}

Twenty-two cryolesions were studied 48 hours and 21 lesions 6 months (Fig. 4) after cryoenergy application. No significant abnormal neointimal proliferation, luminal narrowing, inflammation, or affection of the layers of the adjacent coronary artery were noted. All cryolesions were sharply demarcated. In contrast to the ventricular lesions, all cryolesions at the atrial level exhibited a transmural extension.

\section{Discussion}

Catheter ablation using cryoenergy is considered to be a safe procedure in pediatric patients. In contrast to radiofrequency current application, to the best of our knowledge, no coronary artery obstruction has been reported after cryoablation in humans. In the present experimental study, we did not find any intimal plaque formation or stenosis of the coronary arteries by serial coronary angiographies, intracoronary ultrasound, and histological examinations in young piglets.

In a previous study of our group, ${ }^{2}$ intimal plaque formation of the RCA had been noted in three of eight animals by intracoronary ultrasound 6 months after radiofrequency current application using a comparable experimental setting and animal model. An earlier study of our group had demonstrated narrowing of the lumen of the RCA due to intimal thickening by $25 \%$ and $40 \%$ in two of five piglets after radiofrequency current application. ${ }^{1}$ In contrast to these findings, no intimal plaque formation or stenosis of the coronary artery was noted in the present study after cryoablation neither by selective coronary angiography and intracoronary ultrasound nor by a histological examination.

Aoyama and cowokers ${ }^{20}$ compared cryothermia and radiofrequency current application using an irrigated tip electrode in the coronary sinus within a distance of $2 \mathrm{~mm}$ from the left circumflex coronary artery in adult dogs. Selective coronary angiographies at 5 minutes and 30 minutes post radiofrequency current application identified LCx narrowing at six of 10 ablation sites and at four of nine sites 1-week post radiofrequency current application. Again, no LCx narrowing was noted 5 minutes, 30 minutes, 1 week, or 3 months after cryoablation by selective coronary angiography. In addition, intracoronary ultrasound during or directly after cryoenergy delivery did not show any increased echogenicity, wall thickness, or luminal narrowing of the LCx. In other study, Skanes and coworkers ${ }^{21}$ evaluated the safety and feasibility of cryoablation within the coronary sinus in 14 swines with a mean body weight of $29.8 \mathrm{~kg}$. No arterial spasm or thrombosis during or after cryoablation was noted by coronary angiography. Coronary flow reserve and continuous-flow Doppler monitoring remained unchanged in the animals studied. 


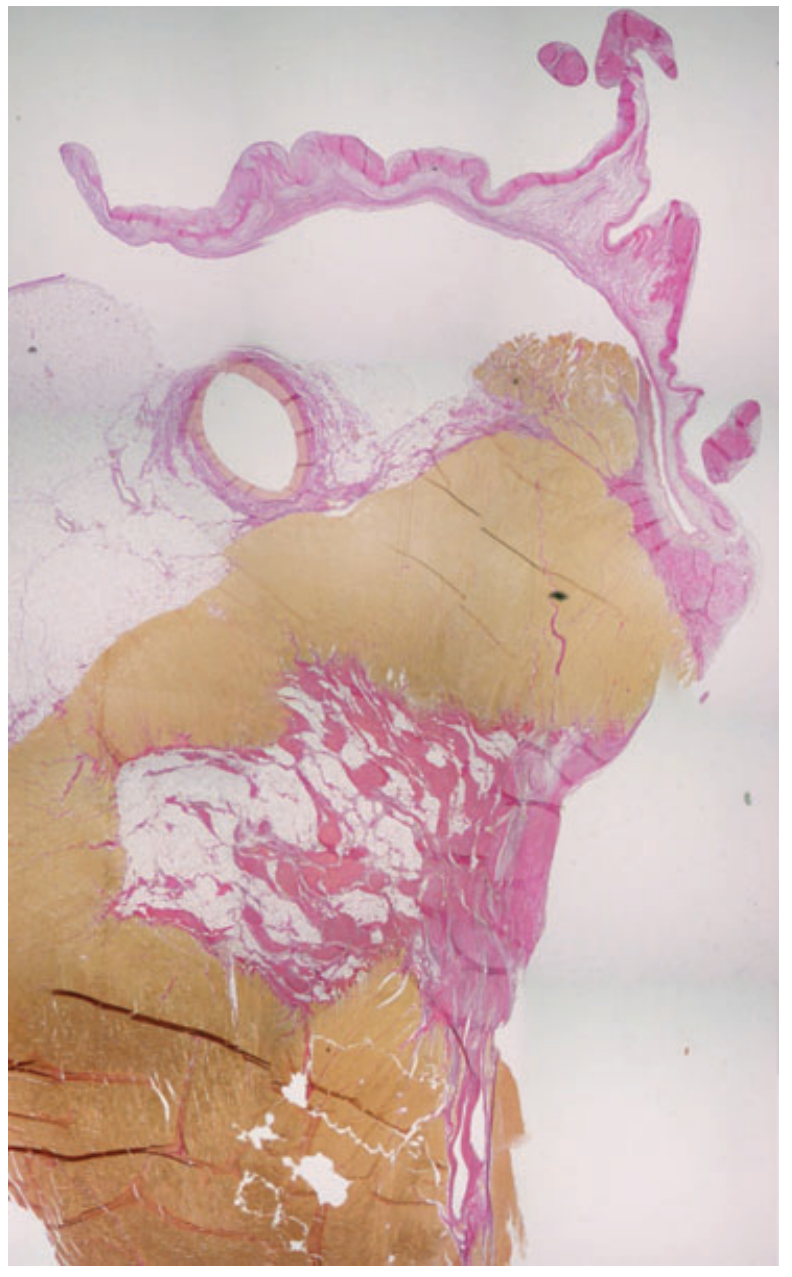

Figure 4. Chronic cryolesion 6 months after energy application at the ventricular aspect of the mitral valve annulus (Elastica Van Gieson staining). The mitral valve is displayed on the top. A sharp demarcated nearly transmural cryolesion is visible. Myocardium is replaced by fibrous tissue in the upper layers and by fat cells in the deeper layers. No obstruction or intima proliferation of the circumflex branch of the left coronary artery is evident.

Additional studies in different animal models $^{22,23}$ demonstrated that epicardial cryolesions directly placed over a coronary artery in the beating heart model or during normothermic cardiopulmonary bypass did not result in any significant coronary artery obstruction. However, in the majority of these studies ${ }^{20,22,23}$ adult animals and/or different locations ${ }^{20-23}$ for energy application had been chosen. Therefore, these results may not represent the situation when cryoenergy is delivered at an immature myocardium. According to the results of the present study, cryoablation seems to be safe with regard to coronary artery obstruction.
Clinical data and animal studies including the present study suggest that cryoablation appears safe with less damage to the coronary artery than with radiofrequency energy. A possible reason may be the smaller volume of cryoenergy lesions and smaller size of affected endocardial surface ${ }^{24}$ using the standard 7-F catheter. This difference may in part be explained by cryoadhesion with tight contact of the catheter tip at the endocardium during every single heart beat resulting in sharply demarcated lesions in contrast to the "brushing effect" during radiofrequency current application. In addition, it may be speculated that coronary flow prevents cryo lesion extension, whereas it does not prevent the extension of the radiofrequency current lesions.

\section{Limitations}

A number of limitations of our experimental study must be considered. First, the present study was performed in a pig model. Despite species differences, we believe that young pigs are the most suitable model for studying the effects of intracardiac radiofrequency current ablation and cryoablation in children due to the similarities in the arrangement and pathologic appearance of coronary arteries in swine and humans. ${ }^{25}$

Second, in the present study cryolesions were performed according to standard protocols ${ }^{16,18,19}$ for the ablation of atrioventricular nodal reentrant tachycardia and accessory pathways in children. It may be speculated that the risk of coronary artery obstruction may increase with longer cryoenergy application time, the use of larger tip electrodes, and/or by lower target temperatures.

\section{Clinical Implications}

In contrast to our previous results using radiofrequency current, we were able to demonstrate that cryoenergy application at an immature myocardium did not result in coronary artery obstruction. Although clinical studies ${ }^{16-18}$ revealed a lower success and higher recurrence rate using cryoenergy for the ablation of accessory pathways compared to radiofrequency current application, we think that cryoenergy should be considered the energy of choice for ablation of tachycardia substrates close to the coronary arteries to minimize the risk of coronary artery obstruction.

\section{Conclusions}

The present study did not show any acute or chronic affection of the coronary arteries after cryoenergy application at growing myocardium by selective coronary angiography, intracoronary ultrasound, and histological examinations in young piglets. 


\section{References}

1. Paul T, Bökenkamp R, Mahnert B, Trappe HJ. Coronary artery involvement early and late after radiofrequency current application in young pigs. Am Heart J 1997; 133:436-440.

2. Sturm M, Hausmann D, Bökenkamp R, Bertram H, Wibbelt G, Paul $T$. Incidence and time course of intimal plaque formation in the right coronary artery after radiofrequency current application detected by intracoronary ultrasound. Z Kardiol 2004; 93:884-889.

3. Bertram H, Bökenkamp R, Peuster M, Hausdorf G, Paul T. Coronary artery stenosis after radiofrequency catheter ablation of accessory atrioventricular pathways in children with Ebstein's malformation. Circulation 2001; 103:538-543.

4. Strobel GG, Trehan S, Compton S, Judd VE, Day RW, Etheridge SP. Successful pediatric stenting of a nonthrombotic coronary occlusion as a complication of radiofrequency catheter ablation. Pacing Clin Electrophysiol 2001; 24:1026-1028.

5. Khanal S, Ribeiro PA, Platt M, Kuhn MA. Right coronary artery occlusion as a complication of accessory pathway ablation in a 12-year-old treated with stenting. Catheter Cardiovasc Interv 1999; 46:59-61.

6. Chatelain P, Zimmermann M, Weber R, Campanini C, Adamec R Acute coronary occlusion secondary to radiofrequency catheter ablation of a left lateral accessory pathway. Eur Heart J 1995; 16:859861.

7. Pons M, Beck L, Leclercq F, Ferriere M, Albat B, Davy JM. Chronic left main coronary artery occlusion: A complication of radiofrequency ablation of idiopathic left ventricular tachycardia. Pacing Clin Electrophysiol 1997; 20:1874-1876.

8. de Paola AA, Leite LR, Arfelli E. Mechanical reperfusion of acute right coronary artery occlusion after radiofrequency catheter ablation and long-term follow-up angiography. J Invasive Cardiol 2003; 15:173-175.

9. Hope EJ, Haigney MC, Calkins H, Resar JR. Left main coronary thrombosis after radiofrequency ablation: Successful treatment with percutaneous transluminal angioplasty. Am Heart J 1995; 129:12171219.

10. Janeira LF. Coronary artery dissection complicating radiofrequency catheter ablation via the retrograde approach. Pacing Clin Electrophysiol 1998; 21:1327-1328.

11. Paul T, Kakavand B, Blaufox AD, Saul JP. Complete occlusion of the left circumflex coronary artery after radiofrequency catheter ablation in an infant. J Cardiovasc Electrophysiol 2003; 14:10041006.

12. Blaufox AD, Saul JP. Acute coronary artery stenosis during slow pathway ablation for atrioventricular nodal reentrant tachycardia in a child. J Cardiovasc Electrophysiol 2004; 15:97-100.

13. Schneider HE, Gonzalez y Gonzalez M, Kriebel T, Paul T. (Abstract). Frequency of coronary artery injury immediately after catheter ablation for supraventricular tachycardias in infants and children. Heart Rhythm 2006; 3(Suppl.):171.
14. Solomon AJ, Tracy CM, Swartz JF, Reagan KM, Karasik PE, Fletscher RD. Effect on coronary artery anatomy of radiofrequency catheter ablation of atrial insertion sites of accessory pathways. J Am Coll Cardiol 1993; 21:1440-1444.

15. Kugler JD, Danford DA, Houston KA, Felix G and the other participating members of the Pediatric Radiofrequency Ablation Registry of the Pediatric Electrophysiology Society. Pediatric radiofrequency catheter ablation registry. Success, fluoroscopy time, and complication rate for supraventricular tachycardia: Comparison of early and recent eras. J Cardiovasc Electrophysiol 2002; 13:336-341.

16. Kirsh JA, Gross GJ, O’Connor S, Hamilton RM; Cryocath International Patient Registry. Transcatheter cryoablation of tachyarrhythmias in children: Initial experience from an international registry. J Am Coll Cardiol 2005; 45:133-136.

17. Miyazaki A, Blaufox AD, Fairbrother DL, Saul JP. Cryo-ablation for septal tachycardia substrates in pediatric patients: Mid-term results. J Am Coll Cardiol 2005; 45:581-588.

18. Kriebel T, Broistedt C, Kroll M, Sigler M, Paul T. Efficacy and safety of cryoenergy in the ablation of atrioventricular reentrant tachycardia substrates in children and adolescents. J Cardiovasc Electrophysiol 2005; 16:960-966.

19. Collins KK, Rhee EK, Kirsh JA, Cannon BC, Fish FA, Dubin AM, Van Hare GF; Pediatric and Congenital Electrophysiology Society's Working Group on Cryoablation. Cryoablation of accessory pathways in the coronary sinus in young patients: A multicenter study from the Pediatric and Congenital Electrophysiology Society's Working Group on Cryoablation. J Cardiovasc Electrophysiol 2007; 18:592-597.

20. Aoyama H, Nakagawa H, Pitha JV, Khammar GS, Chandrasekaran K, Matsudaira K, Yagi T, et al. Comparison of cryothermia and radiofrequency current in safety and efficacy of catheter ablation within the canine coronary sinus close to the left circumflex coronary artery. J Cardiovasc Electrophysiol 2005; 16:1218-1226.

21. Skanes AC, Jones DL, Teefy P, Guiraudon C, Yee R, Krahn, AD, Klein GJ. Safety and feasibility of cryothermal ablation within the mid- and distal coronary sinus. J Cardiovasc Electrophysiol 2004; 15:1319-1323.

22. Holman WL, Ikeshita M, Ungerleider RM, Smith PK, Ideker RE, Cox JL. Cryosurgery for cardiac arrhythmias: Acute and chronic effects on coronary arteries. Am J Cardiol 1983; 51:149-155.

23. Misaki T, Allwork SP, Bentall HH. Long-term effects of cryosurgery in the sheep heart. Cardiovasc Res 1983; 17:61-69.

24. Khairy P, Chauvet P, Lehmann J, Lambert J, Macle L, Tanguay JF, Sirois MG, et al. Lower incidence of thrombus formation with cryoenergy versus radiofrequency catheter ablation. Circulation 2003; 107:2045-2050.

25. McKenzie JE. Swine as a model in cardiovascular research. In: Tumbleson ME, Schook LB (eds.). Advances in Swine in Biomedical Research. 2nd Ed. New York, Plenum Press, 1996, pp. 7-17. 